\title{
DIAGNOSE NUTRICIONAL PARA NITROGÊNIO E POTÁSSIO EM BANANEIRA POR MEIO DO SISTEMA INTEGRADO DE DIAGNOSE E RECOMENDAÇÃO (DRIS) E DE NÍVEIS CRÍTICOS ${ }^{1}$
}

\author{
LUIZ ANTONIO JUNQUEIRA TEIXEIRA², WAGNER RODRIGUES DOS SANTOS ${ }^{3}$, \\ ONDINO CLEANTE BATAGLIA ${ }^{4}$
}

\begin{abstract}
RESUMO - A partir de resultados de um experimento de campo com arranjo fatorial, no qual foi estudada a resposta à aplicação de $\mathrm{N}$ e K em bananeira, em dois ciclos de cultivo, avaliou-se o desempenho do diagnóstico nutricional para esses nutrientes, empregandose o sistema integrado de diagnose e recomendação (DRIS) e o critério de níveis críticos (NC). As situações nas quais se diagnosticou deficiência, foram classificadas como positivas (+), aquelas cujo diagnóstico indicou suficiência, como negativas (-). Em função da resposta na produção de frutos à aplicação de $\mathrm{N}$ ou $\mathrm{K}$, classificaram-se os diagnósticos como verdadeiros (V) ou falsos (F), resultando nas quatro possibilidades V+, V-, F+e F-. Em relação ao N, a eficiência (\%V+e \% V-) dos diagnósticos baseados em NC foi de 48\%; com o DRIS, foi de 69\%. Para K, a eficiência dos diagnósticos feitos a partir do DRIS e NC foi de 63\%. A proporção de diagnósticos de deficiência que se confirmaram com respostas positivas à aplicação de $\mathrm{N}$ em relação aos falsos positivos (\% $\mathrm{V}+\div \% \mathrm{~F}+$ ), foi de 1,50 para o DRIS e de 0,68 para NC. Para os diagnósticos de deficiência de K, essa proporção foi de 1,67 para DRIS e NC. A variação líquida no rendimento (48 casos) decorrente da aplicação de $\mathrm{N}$ associada a diagnósticos corretos foi de $124 \mathrm{t}$ ha ${ }^{-1}$ para o DRIS e de $20 \mathrm{t}$ ha ${ }^{-1}$ para NC. Para K, essa variação foi de $70 \mathrm{t} \mathrm{ha}^{-1}$ para o DRIS e NC.
\end{abstract}

Termos para indexação: análise foliar, banana, nível crítico, nutrição mineral, N , K, Musa spp.

\section{THE N AND K DIAGNOSIS ON BANANA PLANTS USING THE DIAGNOSIS AND RECOMMENDATION INTEGRATED SYSTEM (DRIS) AND CRITICAL VALUE APPROACH}

\begin{abstract}
The Performance of DRIS and critical value approach (CVA) diagnostics were evaluated based on leaf analysis from a field experiment on a factorial design to study the yield response of banana to $\mathrm{N}$ and $\mathrm{K}$ applications during two crop seasons. The situations identified as deficient were classified as positives (+) and those diagnosed as sufficient, were classified as negatives (-). According to the response on yield due the application of $\mathrm{N}$ or $\mathrm{K}$, the diagnostics were classified as true $(\mathrm{V})$ or false $(\mathrm{F})$. Four combinations were possible: $\mathrm{V}+, \mathrm{V}-, \mathrm{F}+$ and $\mathrm{F}-$. The efficiency for $\mathrm{N}$ diagnostics $(\% \mathrm{~V}+$ and $\% \mathrm{~V}-)$ based on the critical value approach was $48 \%$, while using the DRIS it was $69 \%$. The efficiency for K diagnostics was $63 \%$ for both DRIS and CV methods. The relation between deficient diagnostics confirmed by positive response on yield under application of the nutrient and false positive cases $(\% \mathrm{~V}+\div \% \mathrm{~F}+)$ for $\mathrm{N}$ was 1.5 for the DRIS and 0.68 for CVA. For the $\mathrm{K}$ deficiency diagnostics, the value of this relation was 1.67 for both methods. The net yield derived from $\mathrm{N}$ application based on correct diagnostics (48 cases) was $124 \mathrm{Mg} \mathrm{ha}^{-1}$ for DRIS and $20 \mathrm{Mg} \mathrm{ha}^{-1}$ for the CVA. For $\mathrm{K}$, this variation was $70 \mathrm{Mg} \mathrm{ha}^{-1}$ for the DRIS and the CVA.
\end{abstract}

Index terms: Musa spp., plant analysis, critical level, mineral nutrition, nitrogen, potassium.

\section{INTRODUÇÃO}

O diagnóstico nutricional elaborado a partir de resultados de análise de tecido vegetal constitui-se num instrumento importante para detectar eventuais deficiências e auxiliar no processo de recomendação de fertilizantes. Entretanto, diagnosticar deficiência nutricional exige, além de método adequado para amostragem e análise de tecido vegetal, o estabelecimento de critérios para a interpretação dos resultados (Munson \& Nelson, 1973). Martin-Prével (1984) demonstrou a importância da análise foliar para o monitoramento do estado nutricional em bananeira e destacou os esforços para a padronização dos procedimentos de amostragem.
A bananeira caracteriza-se por sua elevada exigência de $\mathrm{N}$ e $\mathrm{K}$ e necessidade de um fornecimento equilibrado desses nutrientes. Na elaboração de diagnósticos nutricionais, usualmente, comparam-se os resultados de análise foliar com valorespadrão (concentrações críticas ou faixas de suficiência), admitindo-se que nutrientes, cuja concentração seja inferior a esses limites, provavelmente estejam limitando o crescimento, rendimento ou qualidade dos frutos (Martin-Prével, 1990; Lahav, 1995). Lahav \& Turner (1983) e Lahav (1995) estabeleceram níveis críticos (NC) para as concentrações de elementos em tecido foliar de bananeira com base em resultados de vários experimentos com bananeira realizados em diversas condições de cultivo. Segundo os autores, esses padrões têm-se mostrado consistentes e

1 (Trabalho 132/2001). Recebido: 11/07/2001. Aceito para publicação: 14/03/2002.

2 Pesquisador do Centro de Fruticultura/IAC. Cx. Postal 28. 13001-970, Campinas, SP. teixeira@iac.br

3 Bolsista. Centro de Solos e Recursos Agroambientais/IAC.

4 Pesquisador do Centro de Solos e Recursos Agroambientais/IAC.

Rev. Bras. Frutic., Jaboticabal - SP, v. 24, n. 2, p. 530-535, agosto 2002 
úteis para diagnosticar deficiências.

Uma abordagem alternativa, possivelmente mais eficiente para revelar desequilíbrios nutricionais e mais robusta em relação a variações na amostragem (idade da planta, época, etc.), é o sistema integrado de diagnose e recomendação - DRIS, no qual, são utilizadas relações entre as concentrações foliares dos nutrientes para interpretar os resultados de análise de tecido (Beaufils, 1973; Walworth \& Sumner, 1987). Angeles et al. (1993), ao analisar resultados de experimentos de adubação em bananeira, constataram limitações em diagnósticos nutricionais estabelecidos por nutrientes individualmente, sem considerar as relações de proporção entre eles. A partir de um banco de dados com informações de composição foliar obtidas em experimentos (915 observações) realizados em diversas condições de ambiente e manejo da cultura, esses autores propuseram padrões (normas) DRIS preliminares para bananeira. Por serem normas preliminares, Angeles et al. (1993) recomendaram em sua conclusão que fossem realizadas validações em experimentos do tipo fatorial, para subsidiar posssíveis ajustes.

Beverly \& Hallmark (1992) demonstraram a necessidade de testar a qualidade dos diversos procedimentos para interpretação de análise de tecido, a fim de verificar sua utilidade como guia para a recomendação de fertilizantes e propuseram um método para realizar tal avaliação. Esse método possibilita quantificar o desempenho de critérios para diagnóstico nutricional (DRIS, NC, faixa de suficiência, entre outros) mediante comparação entre o previsto (situação de deficiência ou suficiência) e a resposta da planta à aplicação de fertilizantes.

O objetivo desse trabalho foi avaliar o desempenho de diagnósticos nutricionais para nitrogênio e potássio em bananeira estabelecidos a partir do critério de níveis críticos de $\mathrm{N}$ e $\mathrm{K}$ e do sistema integrado de diagnose e recomendação (DRIS).

\section{MATERIAL E MÉTODOS}

Diagnósticos nutricionais para $\mathrm{N}$ e K elaborados a partir de NC e DRIS foram confrontados com a resposta de rendimento das plantas à aplicação de fertilizantes nitrogenados e potássicos em dois ciclos de produção de bananeiras- 'Nanicão' (Musa AAA, subgrupo Cavendish). Dados de amostragem foliar (limbo da folha três de plantas com cachos emitidos), bem como de produção, foram obtidos a partir de um experimento de campo descrito por Teixeira (2000). Os tratamentos foram arranjados em parcelas subdivididas, sendo as parcelas principais constituídas por dois regimes hídricos: irrigado (microaspersão) e sequeiro, e as subparcelas, pelo fatorial com quatro doses de nitrogênio $\left(0 ; 200 ; 400\right.$ e $\left.800 \mathrm{~kg} \mathrm{ha}^{-1} \mathrm{de} \mathrm{N}\right)$ e quatro de potássio $(0 ; 300 ; 600 \mathrm{e}$ $900 \mathrm{~kg} \mathrm{ha}^{-1}$ de $\mathrm{K}_{2} \mathrm{O}$ ). Os adubos, nitrato de amônio e cloreto de potássio (fontes de $\mathrm{N}$ e K, respectivamente), foram aplicados na superfície do solo, sem incorporação, fracionados em quatro vezes durante a estação das chuvas. Resultados da análise química do solo, em amostragem realizada na época da instalação do experimento, encontram-se na Tabela 1.

Tecido foliar foi amostrado de acordo com as recomendações de Martin-Prével (1984) e consistiu na coleta da porção central do limbo da terceira folha, contando-se a partir do ápice, na época em que a inflorescência apresentava duas ou três pencas masculinas abertas. As amostras foram processadas e analisa- das quanto aos teores de N, P, K, Ca e Mg de acordo com Bataglia et al. (1983).

Para a elaboração dos diagnósticos nutricionais baseados na comparação dos teores de $\mathrm{N}$ e $\mathrm{K}$ foliares com os padrões para níveis críticos desses nutrientes em bananeira, empregaram-se os valores apresentados por Lahav (1995). Definiu-se como diagnóstico de deficiência, valores de concentração foliar de $\mathrm{N}$ ou $\mathrm{K}$ inferiores aos padrões expressos na Tabela 2.

Os índices para o sistema integrado de diagnose e recomendação (DRIS) foram calculados a partir das normas publicadas por Angeles et al. (1993). Para o cálculo das funções intermediárias (relações entre nutrientes), empregou-se a fórmula proposta por Jones (1981):

$$
f(X / Y)=(X / Y-x / y) . K / s
$$

onde: $\mathrm{X} / \mathrm{Y}=$ valor da relação entre as concentrações dos dois nutrientes na planta sob diagnóstico; conforme os nutrientes envolvidos, essa relação pode ser uma razão ou produto (Tabela 2); $\mathrm{x} / \mathrm{y}=$ valor ótimo ou norma para a relação entre os nutrientes (Tabela 2); K = 10 (constante de valor arbitrário); s = desviopadrão da relação x/y (norma) na população de referência (Tabela 2).

Os índices para cada nutriente foram calculados de acordo com Walworth \& Sumner (1987). Diagnosticou-se deficiência nutricional de $\mathrm{N}$ ou $\mathrm{K}$ nas situações em que os índices DRIS para esses nutrientes eram menores que zero, como apresentado em Jones (1981).

Empregou-se o procedimento proposto por Beverly \& Hallmark (1992) para avaliar o desempenho dos diagnósticos nutricionais para $\mathrm{Ne} \mathrm{K}$ realizados com base em DRIS e NC, cujos critérios são apresentados na Tabela 3. Os diagnósticos foram confrontados com as respostas das plantas à aplicação de $\mathrm{N} \mathrm{e} \mathrm{K}$ observadas no experimento fatorial. Selecionaram-se 48 casos (quatro doses de $\mathrm{N}$ ou $\mathrm{K}$, três incrementos de $\mathrm{N}$ ou $\mathrm{K}$, duas safras e dois regimes hídricos), nos quais a dose do nutriente em questão crescia, mantendo-se as demais condições constantes. As situações nas quais se diagnosticou deficiência, foram classificadas como positivas (+), aquelas cujo diagnóstico indicou suficiência, como negativas (-). Em função da resposta na produção de frutos à aplicação de $\mathrm{N}$ ou $\mathrm{K}$, classificaram-se os diagnósticos como verdadeiros (V) ou falsos (F), resultando nas quatro possibilidades $\mathrm{V}+, \mathrm{V}-, \mathrm{F}+$ e F-. A eficiência dos diagnósticos foi estimada pela soma dos percentuais de diagnósticos verdadeiros (\%V+e \%V-). Calculou-se outro indicador de qualidade definido pela proporção entre diagnósticos verdadeiros e falsos para as situações nas quais se diagnosticou deficiência nutricional $(\% \mathrm{~V}+\% \mathrm{~F}+)$. Por fim, foi estimada a variação no rendimento possivelmente associada ao método de diagnóstico ( $\mathrm{S} \mathrm{d}(\mathrm{Y})$ ); para tanto, incrementos de rendimento com diagnósticos V+e decréscimos com V-foram somados (respostas previstas pelo diagnóstico), decréscimos no rendimento com $\mathrm{F}+\mathrm{e}$ incrementos com F- (respostas não previstas pelo diagnóstico) foram subtraídos.

\section{RESULTADOS E DISCUSSÃO}

$\mathrm{Na}$ Tabela 4, são apresentados diagnósticos nutricionais para nitrogênio e potássio elaborados com as médias dos teores foliares obtidos em cada uma das quatro doses de 
N e K. A aplicação de fertilizantes nitrogenados e potássicos determinou incrementos nos teores foliares de $\mathrm{N}$ e K, assim como nos índices DRIS para esses nutrientes, mostrando que esses dois indicadores foram sensíveis à maior disponibilidade. Para nitrogênio, foram diagnosticadas deficiências, segundo o critério de NC no primeiro ciclo de cultivo, que não corresponderam à resposta das plantas à aplicação de $\mathrm{N}$, que foi negativa ou nula (Tabela 5). Com o DRIS, nesse ciclo, os valores do índice para $\mathrm{N}$ sempre foram positivos, indicando suficiência ou excesso, o que está de acordo com as respostas apresentadas na Tabela 5.

Na segunda safra, pelo critério do NCs diagnosticou-se deficiência de $\mathrm{N}$ independentemente da adubação nitrogenada. Tanto o critério dos NC como o DRIS indicaram deficiência de potássio independentemente da adubação e irrigação nos dois ciclos de cultivo (Tabela 4). Entretanto, pelo DRIS, detecta-se que o $\mathrm{K}$ foi o nutriente mais limitante no primeiro ciclo, especialmente sem irrigação, o que está coerente com a resposta positiva (Tabela 5) à adubação potássica nessa situação.

$\mathrm{O}$ desempenho dos diagnósticos nutricionais para $\mathrm{N}$ e $\mathrm{K}$, empregando-se o DRIS, e o critério dos NCs em bananeira, avaliado segundo critérios de Beverly \& Hallmark (1992), é apresentado na Tabela 6.

Para N, o desempenho do DRIS foi superior ao critério de NC. A eficiência do DRIS (69\%) foi maior que a do NC (48\%) devido ao elevado percentual de diagnósticos de deficiência que não se confirmaram com aplicação de fertilizante nitrogenado $(\mathrm{F}+)$. A baixa eficiência do critério do NC para diagnosticar ne- cessidade de aplicação de $\mathrm{N}$ implicou que, em quase metade dos casos (46\%), fossem indicadas aplicações que, além de desnecessárias, causaram queda no rendimento. Entre as situações nas quais se diagnosticou deficiência de $\mathrm{N}$ por meio do DRIS, houve mais diagnósticos verdadeiros do que falsos, na proporção de 1,5:1. Com NC, entre os diagnósticos de deficiência, constatou-se que a maior parte era falsa. Por fim, as variações no rendimento decorrentes da aplicação de $\mathrm{N}$ associadas aos diagnósticos foram superiores com o DRIS. O somatório das variações de rendimento, nos 48 casos estudados, também indicou que o desempenho do DRIS foi superior ao critério de NC. Melhor desempenho do DRIS, em relação ao NC, para diagnósticos com $\mathrm{N}$ em tomateiro foi relatado por Caron et al. (1991), que o relacionaram com a maior sensibilidade do DRIS para detectar situações de desequilíbrio por excesso de $\mathrm{N}$.

Nos diagnósticos para $\mathrm{K}$, tanto o critério dos $\mathrm{NC}$, como o DRIS, apresentaram desempenho equivalente. A eficiência dos diagnósticos foi superior a 50\%, e a proporção entre os diagnósticos verdadeiros e falsos para situações de deficiência foi maior do que um (Tabela 6). Os dois métodos de diagnósticos indicaram deficiência de $\mathrm{K}$ em todos os casos estudados.

Na prática, a decisão de aplicar fertilizante é tomada levando em consideração outros aspectos, como histórico da área, análise de solo, potencial da cultura, etc., além do diagnóstico baseado na análise de tecido foliar. Entretanto, valores de eficiência menores que $50 \%$ são inaceitáveis para qualquer método de diagnóstico. Segundo Beverly \& Hallmark (1992), caso a

TABELA 1 - Análise química do solo da área experimental (Teixeira, 2000)

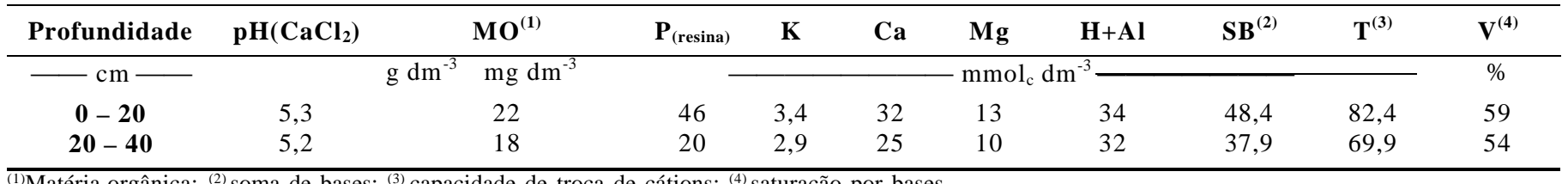

${ }^{(1)}$ Matéria orgânica; ${ }^{(2)}$ soma de bases; ${ }^{(3)}$ capacidade de troca de cátions; ${ }^{(4)}$ saturação por bases.

TABELA 2 - Normas DRIS com desvio-padrão e níveis críticos para concentrações foliares de nutrientes em bananeira

\begin{tabular}{cccc}
\hline \multirow{2}{*}{$\begin{array}{c}\text { Elemento ou } \\
\text { relação }\end{array}$} & Norma & DRIS $^{(\mathbf{1})}$ & Nível crítico \\
\cline { 2 - 3 } & & & Desvio-padrão $^{(2)}$ \\
$\mathbf{N}$ & $3,04 \%$ & 0,2326 & $\mathrm{~g} \mathrm{~kg}^{-1}-$ \\
$\mathbf{P}$ & $0,23 \%$ & 0,0262 & 26,0 \\
$\mathbf{K}$ & $4,49 \%$ & 0,4804 & 30,0 \\
$\mathbf{C a}$ & $0,80 \%$ & 0,2628 & 5,0 \\
$\mathbf{M g}$ & $0,41 \%$ & 0,0994 & 3,0 \\
$\mathbf{P} / \mathbf{N}$ & 0,07 & 0,0094 & - \\
$\mathbf{K} / \mathbf{N}$ & 1,49 & 0,2135 & - \\
$\mathbf{K} / \mathbf{P}$ & 19,79 & 2,7884 & - \\
$\mathbf{C a} \times \mathbf{N}$ & 2,43 & 0,9027 & - \\
$\mathbf{M g} \times \mathbf{N}$ & 1,23 & 0,2839 & - \\
$\mathbf{C a} \times \mathbf{P}$ & 0,18 & 0,0588 & - \\
$\mathbf{M g} \times \mathbf{P}$ & 0,09 & 0,0216 & - \\
$\mathbf{M g} \times \mathbf{K}$ & 1,82 & 0,4708 & - \\
$\mathbf{C a} \times \mathbf{K}$ & 3,51 & 1,0614 & - \\
$\mathbf{M g} / \mathbf{C a}$ & 0,59 & 0,2694 & \\
\hline
\end{tabular}

(1) Angeles et al. (1993); (2) Lahav (1995). 
TABELA 3 - Cálculo, amplitude e valores aceitáveis dos critérios para avaliação do desempenho de diagnósticos nutricionais (adaptado de Beverly \& Hallmark, 1992)

\begin{tabular}{cccc}
\hline Critério & Cálculo & A mplitude & Valores aceitáveis \\
\hline Eficiência & $(\% \mathrm{~V}+)+(\% \mathrm{~V}-)$ & 0 a 100 & $>50$ \\
$\mathbf{V} \div \mathbf{F}$ & $(\% \mathrm{~V}+) \div(\% \mathrm{~F}+)$ & 0 a 100 & $>1$ \\
$\Sigma \mathbf{d}(\mathrm{Y})$ & $[\mathrm{d}(\mathrm{Y}) \mathrm{V}+]-[\mathrm{d}(\mathrm{Y}) \mathrm{V}-]-]_{-}^{(1)}$ & $-\propto$ a $+\propto$ & $>0$ \\
& {$[\mathrm{~d}(\mathrm{Y}) \mathrm{F}-]+[\mathrm{d}(\mathrm{Y}) \mathrm{F}+]$} & & \\
\hline
\end{tabular}

(1) $[\mathrm{d}(\mathrm{Y}) \mathrm{V}+],[\mathrm{d}(\mathrm{Y}) \mathrm{V}-],[\mathrm{d}(\mathrm{Y}) \mathrm{F}-]$ e $[\mathrm{d}(\mathrm{Y}) \mathrm{F}+]$ = respostas à aplicação de fertilizantes associadas aos diagnósticos $\mathrm{V}+, \mathrm{V}-, \mathrm{F}-$ e F+, respectivamente.

TABELA 4 - Diagnóstico nutricional para bananeira num experimento fatorial $\mathrm{N} K$ por meio do critério de níveis críticos e do DRIS

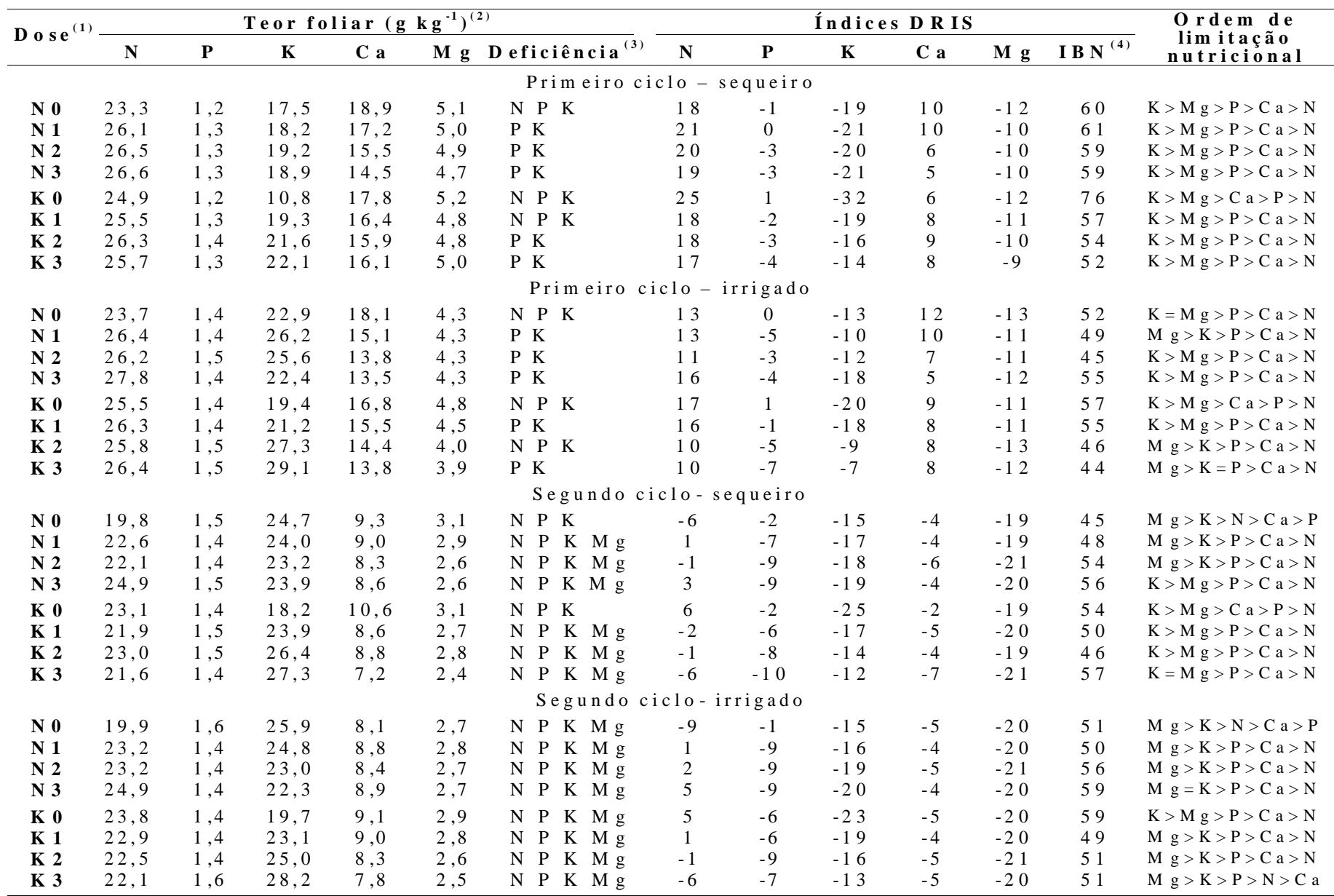

(1) N0, N1, N2 e N3 = 0; 200; 400 e $800 \mathrm{~kg} \mathrm{ha}^{-1}$ de N, respectivamente; K0, K1, K2 e K3 = 0; 300; 600 e $900 \mathrm{~kg} \mathrm{ha}^{-1}$ de $\mathrm{K}_{2} \mathrm{O}$, respectivamente; ${ }^{(2)}$ teores no limbo da folha três de plantas com cachos recém-emitidos; ${ }^{(3)}$ deficiência $=$ teor foliar $<\mathrm{NC}$ da Tabela 2 ; ${ }^{(4)}$ IBN=índice de balanço nutricional definido pelo somatório dos índices (em módulo) de cada nutriente (ótimo=0).

TABELA 5 - Efeito da aplicação de $\mathrm{Ne} \mathrm{K}$ (doses em $\mathrm{kg} \mathrm{ha}^{-1}$ de $\mathrm{Ne} \mathrm{K}_{2} \mathrm{O}$, respectivamente) sobre o rendimento de frutos (t ha-1), sob duas condições de irrigação, em dois ciclos de produção ${ }^{(1)}$

\begin{tabular}{|c|c|c|c|}
\hline Nutriente & Regime hídrico & $\mathbf{E} \mathbf{q} \mathbf{u}$ a ç ã o & $\mathbf{R}^{2}$ \\
\hline \multicolumn{4}{|c|}{ Primeiro ciclo } \\
\hline $\mathbf{N}$ & $\begin{array}{l}\text { Sequeiro } \\
\text { Irrigado }\end{array}$ & $\begin{array}{l}\mathrm{Y}=\mathrm{f}(\mathrm{N}) \mathrm{n} \cdot \mathrm{s} . \\
\mathrm{Y}=36,01-3,44 \times 10^{-2} \mathrm{~N}+2,62 \times 10^{-5} \mathrm{~N}^{2}\end{array}$ & $0, \overline{9} 1$ \\
\hline $\mathbf{K}$ & $\begin{array}{l}\text { Sequeiro } \\
\text { Irrigado }\end{array}$ & $\begin{aligned} \mathrm{Y}= & 16,62+1,69 \times 10^{-2} \mathrm{~K}-1,12 \times 10^{-5} \mathrm{~K}^{2} \\
\mathrm{Y}= & \mathrm{f}(\mathrm{K}) \mathrm{n} \cdot \mathrm{s} . \\
& \text { Segundo ciclo }\end{aligned}$ & $\begin{array}{c}0,88 \\
-\end{array}$ \\
\hline $\mathbf{N}$ & $\begin{array}{l}\text { Sequeiro } \\
\text { Irrigado }\end{array}$ & $\begin{array}{l}\mathrm{Y}=30,67+3,15 \times 10^{-2} \mathrm{~N}-3,59 \times 10^{-5} \mathrm{~N}^{2} \\
\mathrm{Y}=36,47+7,07 \times 10^{-2} \mathrm{~N}-7,09 \times 10^{-5} \mathrm{~N}^{2}\end{array}$ & $\begin{array}{l}0,62 \\
0,98\end{array}$ \\
\hline $\mathbf{K}$ & $\begin{array}{l}\text { Sequeiro } \\
\text { Irrigado }\end{array}$ & $\begin{array}{l}Y=f(K) n \cdot s \\
Y=f(K) n \cdot s\end{array}$ & $\begin{array}{l}- \\
-\end{array}$ \\
\hline
\end{tabular}

\footnotetext{
(1) Tabela montada a partir dos resultados apresentados em Teixeira (2000).
} 
TABELA 6 - Desempenho de diagnósticos nutricionais para N e K em bananeira com base em eficiência, incidência e variações no rendimento associadas a diagnósticos verdadeiros e falsos

\begin{tabular}{|c|c|c|c|c|c|c|c|c|}
\hline \multirow{2}{*}{ Método } & \multirow{2}{*}{ Nutriente } & \multicolumn{4}{|c|}{ Diagnósticos ${ }^{(1)}$} & \multirow{2}{*}{ Eficiência ${ }^{(2)}$} & \multirow{2}{*}{$\mathbf{V} \div \mathbf{F}^{(3)}$} & \multirow{2}{*}{$\Sigma \mathrm{d}(\mathbf{Y})^{(4)}$} \\
\hline & & $\mathrm{V}+$ & $\mathbf{F}+$ & $\mathrm{V}-$ & F - & & & \\
\hline & & & - - & $-\%$ & - & -- & & $\mathrm{tha}^{-1}$ \\
\hline $\operatorname{DRIS}{ }^{(5)}$ & $\mathrm{N}$ & 19 & 12 & 50 & 19 & 69 & 1,50 & 124 \\
\hline $\mathrm{NC}^{(6)}$ & $\mathrm{N}$ & 31 & 46 & 17 & 6 & 48 & 0,68 & 20 \\
\hline DRIS & $\mathrm{K}$ & 63 & 37 & 0 & 0 & 63 & 1,67 & 70 \\
\hline N C & $\mathrm{K}$ & 63 & 37 & 0 & 0 & 63 & 1,67 & 70 \\
\hline
\end{tabular}

(1) $\mathrm{V}+=$ deficiência verdadeira, $\mathrm{F}+=$ deficiência falsa, $\mathrm{V}-=$ suficiência verdadeira, $\mathrm{F}-\mathrm{suficiência} \mathrm{falsa;}{ }^{(2)}$ eficiência $=(\% \mathrm{~V}+)+(\% \mathrm{~V}-) ;{ }^{(3)} \mathrm{V} \div \mathrm{F}=(\% \mathrm{~V}+) \div(\% \mathrm{~F}+)$;

(4) somatório das variações no rendimento associadas aos diagnósticos (48 casos); ${ }^{(5)}$ normas DRIS de Angeles et al. (1993); ${ }^{(6)}$ níveis críticos definidos por Lahav (1995).

eficiência seja inferior a 50\% (mais da metade dos diagnósticos não confirmados por respostas à aplicação de fertilizantes), prever a necessidade de fertilizante, jogando cara ou coroa com uma moeda não viciada, teria maior probabilidade de acerto. Angeles et al. (1993) observaram que o DRIS foi superior ao critério dos NCs no acerto de diagnósticos nutricionais para N, P e K em bananeira. Com laranjeira, Beverly et al. (1984) concluíram que os diagnósticos por meio de NC e do DRIS foram equivalentes. Entretanto, observaram que o DRIS foi sensível ao tipo e idade do tecido amostrado, o que contraria a maioria da literatura. $\mathrm{O}$ DRIS possibilitou fazer diagnósticos para N, P e K com maior percentual de acertos do que o critério dos NCs em abacaxizeiro (Angeles et al., 1990).

A interpretação para bananeira dos teores de nutrientes com base em NC preestabelecidos requer alguns cuidados. Além da amostragem padronizada para a cultura (época e tipo de tecido), devem-se considerar possíveis variações devidas ao ambiente e variedades (Martin-Prével, 1977; Lahav \& Turner, 1985; Turner \& Lahav, 1985). Por outro lado, o emprego do DRIS, por ser um critério menos sensível a variações na amostragem (época, idade da planta, etc.), poderia ser mais flexível. Esse aspecto, de certa forma, foi confirmado neste trabalho, pois foi possível obter diagnósticos com base no DRIS para N e K com qualidade equivalente ou superior àqueles baseados em NC, mesmo utilizando-se de normas DRIS preliminares e obtidas em condições bem diversas do experimento que originou esta validação.

\section{CONCLUSÕES}

1.A eficiência dos diagnósticos com DRIS para nitrogênio foi superior à daqueles baseados no critério de nível crítico, assim como a proporção de diagnósticos de deficiência que se confirmaram com respostas positivas à aplicação de $\mathrm{N}$.

2.Para potássio, tanto o DRIS como o critério de nível crítico apresentaram desempenhos semelhantes.

3. A variação líquida no rendimento decorrente da aplicação de $\mathrm{N}$ associada a diagnósticos corretos foi superior para o DRIS em relação ao critério de nível crítico. Para potássio, essa variação foi a mesma para os dois critérios de diagnóstico.

\section{REFERÊNCIAS BIBLIOGRÁFICAS}

ANGELES, D.E.; SUMNER, M.E.; LAHAV, E. Preliminary DRIS norms for banana. Journal of Plant Nutrition, New York, v. 16, p.1059-70, 1993.

ANGELES, D.E.; SUMNER, M.E.; BARBOUR, N.W. Preliminary nitrogen, phosphorus, and potassium DRIS norms for pineapple. HortScience, Alexandria, v.25, p.652-5, 1990.

BATAGLIA, O.C.; FURLANI, A.M.C.; TEIXEIRA, J.P.F.; FURLANI, P.R.; GALLO, J.R. Métodos de análise química de plantas. Campinas: IAC, 1983. 48p. (Boletim Técnico, 78).

BEAUFILS, E.R. Diagnosis and recommendation integrated system (DRIS). S. Africa: University of Natal, 1973. 132p. (Soil Science Bull., 1).

BEVERLY, R.B.; HALLMARK, W.B. Prescient diagnostic analysis: a proposed new approach to evaluating plant nutrient diagnostic methods. Communications in Soil Science and Plant Analysis, New York, v. 23, p.2633-40, 1992.

BEVERLY, R.B.; STARK, J.C., OJALA, J.C.; EMBLETON, T.W. Nutrient diagnosis of 'Valencia' oranges by DRIS. Journal of American Society of Horticultural Science, St. Joseph, v.109, p.649-54, 1984.

CARON, J.; PARENT, L.E.; GOSSELIN, A. Effect of nitrogen and salinity levels in the nutrient solution on the DRIS diagnosis of greenhouse tomato. Communications in Soil Science and Plant Analysis, New York, v. 22, p.879-92, 1991.

JONES, C.A. Proposed modifications of the diagnosis and recommendation integrated system (DRIS) for interpreting plant analyses. Communications in Soil Science and Plant Analysis, New York, v.12, p.785-94, 1981.

LAHAV, E. Banana nutrition. In: GOWEN, S. (Ed.) Bananas and plantains. London: Chapman \& Hall, 1995. p.258-316.

LAHAV, E.; TURNER, D.W. Temperature influences the composition of diagnostic samples used to assess the nutrient status of banana plants. Scientia Horticulturae, Amsterdam, v.27, p.27583,1985 .

LAHAV, E.; TURNER, D.W. Banana nutrition. Berna: IPI, 1983. 62p. (IPI-Bulletin, 7).

MARTIN-PRÉVEL, P. Echantillonnage du bananier pour l'analyse

Rev. Bras. Frutic., Jaboticabal - SP, v. 24, n. 2, p. 530-535, agosto 2002 
foliaire: Conséquences des différences de techniques. Fruits, Paris, v.32, p.151-66, 1977.

MARTIN-PRÉVEL, P. Bananier. In: MARTIN-PRÉVEL, P., GAGNARD, J., GAUTIER, P. (Eds.) L'analyse végétale dans le contrôle de l'alimentation des plantes tempérées et tropicales. Paris: Tec\&Doc, 1984. p.715-51.

MARTIN-PRÉVEL, P. Past, present and future of tropical fruit nutrition with special reference to banana nutrition. Acta Horticulturae, Leuven, v.275, p. 523-34, 1990.

MUNSON, R.D.; NELSON, W.L. Principles and practices in plant analysis. In: WALSH, L.M.; BEATON, J.D. (Ed.)Soil testing and plant analysis. Madison: SSSA, 1973. p.223-48.
TEIXEIRA, L.A.J. Adubação nitrogenada e potássica em bananeira 'Nanicão' (Musa AAA subgrupo Cavendish) sob duas condições de irrigação. Jaboticabal, 2000. 132f. Tese (Doutorado) Faculdade de Ciências Agrárias e Veterinárias, Universidade Estadual Paulista, Jaboticabal, 2000.

TURNER, D.W.; LAHAV, E. Temperature influences nutrient absorption and uptake rates of bananas grown in controlled environments. Scientia Horticulturae, Amsterdam, v.26, p.311-22, 1985.

WALWORTH, J.L.; SUMNER, M.E. The diagnosis and recommendation integrated system (DRIS). In: Advances in Soil Science. New York: Springer-Verlag, 1987. v.6, p.149-88. 